\title{
Normal ESR, CRP and Platelet Count in Giant Cell Arteritis and Polymyalgia Rheumatica: A Diagnostic Conundrum
}

\author{
Abdullahi Elsheikh Mahgoub ${ }^{1}$, Sophia Tessema², Rasha Nakhleh ${ }^{3}$ \\ ${ }^{1}$ Department of Internal Medicine, Michigan State University at Hurley Medical Center, Flint, MI, USA \\ ${ }^{2}$ Department of Neurology, Wayne State University, Detroit, MI, USA \\ ${ }^{3}$ Division of General Internal Medicine and Geriatrics, Oregon Health and Science University, Portland, OR, USA
}

Doi: 10.12890/2022_003192 - European Journal of Case Reports in Internal Medicine - ๔ EFIM 2022

Received: 15/01/2022

Accepted: 19/01/2022

Published: $21 / 02 / 2022$

\begin{abstract}
How to cite this article: Mahgoub AE, Tessema S, Nakhleh R. Normal ESR, CRP and platelet count in giant cell arteritis and polymyalgia rheumatica: a diagnostic conudrum. EJCRIM 2022;9: doi:10.12890/2022_003192.
\end{abstract}

Conflicts of Interests: The authors declare there are no competing interests.

This article is licensed under a Commons Attribution Non-Commercial 4.0 License

\section{ABSTRACT}

Giant cell arteritis (GCA) and polymyalgia rheumatica (PMR) are both rheumatological diseases of the elderly with a strong association with each other and which rarely present with normal inflammatory markers. Here we present the case of a 61-year-old Caucasian woman who had typical symptoms of both diseases. At the time of presentation, her blood work showed normal inflammatory markers, but because of the high clinical suspicion for GCA, a temporal artery biopsy was done which was positive for giant cells and disruption of the internal elastic lamina. Our patient responded very well to treatment with oral steroids and steroid-sparing medication and was able to return to her normal life without experiencing any complications of the disease. By sharing our case, we aim to increase the awareness of medical personnel regarding the importance of focusing on the clinical presentation as well as the laboratory and pathological aspects of diagnosing GCA and PMR.

\section{LEARNING POINTS}

- Normal levels of inflammatory markers like C-reactive protein and the erythrocyte sedimentation rate do not rule out the diagnosis of giant cell arteritis (GCA).

- We aim to increase clinician recognition and awareness of other parameters, particularly the clinical presentation, that should be considered when diagnosing GCA or polymyalgia rheumatica.

- If the index of suspicion for the disease is high, it is crucial to start treatment as early as possible for better management and to avoid harmful complications.

\section{KEYWORDS}

Giant cell arteritis, polymyalgia rheumatica, inflammatory markers, ESR, CRP, vasculitis

\section{INTRODUCTION}

Temporal arteritis (TA) or giant cell arteritis (GCA) is a form of vasculitis that affects large and medium-sized blood vessels; it is almost exclusively a disease of the elderly ${ }^{[1]}$. Presentation is usually with a wide array of symptoms like headache, visual disturbances and jaw claudication. GCA has a strong association with polymyalgia rheumatica (PMR), which is one of the most common inflammatory rheumatic diseases of the elderly and rarely occurs in people below 50 years of age ${ }^{[2]}$. GCA symptoms can overlap with those of other rheumatological diseases and the diagnosis can be challenging. However, the American College of Rheumatology (ARC) has developed criteria that are 
widely used for diagnosing GCA and consist of five components: age at disease onset, new onset of localized headache, temporal artery abnormality, elevated erythrocyte sedimentation rate (ESR) and abnormal temporal artery biopsy (TAB). At least three of these five criteria should be met for diagnosis ${ }^{[3]}$. Other guidelines include the Swedish Society of Rheumatology 2018 recommendations for the investigation, treatment and follow-up of GCA, which included new components like C-reactive protein (CRP) CRP $\geq 10$ mg/l and imaging diagnostics using ultrasonography, MRI and/or FDG-PET ${ }^{[4]}$.

The American College of Rheumatology and the European League against Rheumatism have developed provisional classification criteria for PMR: patients aged $\geq 50$ years old presenting with bilateral shoulder pain, not better explained by an alternative pathology, can be classified as having PMR in the presence of morning stiffness for $>45 \mathrm{~min}$, elevated CRP and/or erythrocyte sedimentation rate (ESR), and new hip pain ${ }^{[5]}$.

This case highlights the presence of GCA and PMR with normal inflammatory markers.

\section{CASE DESCRIPTION}

Our patient was a 61-year-old Caucasian woman without a significant medical history. She presented to her family physician with generalized fatigue in addition to pain and stiffness around both hips and shoulders. She also had difficulty standing from a seated position and raising her arms above her shoulders. She denied fever or visual impairment but reported some headaches. She had never smoked cigarettes, drank alcohol occasionally and had no history of illicit drug use. Upon initial evaluation, she was haemodynamically stable and afebrile and was saturating well on ambient air. Her physical examination was significant for proximal myopathy involving both upper and lower extremities. The temporal artery was not palpable. Sensation, coordination and reflexes were intact during neurological examination.

Extensive work-up was done including a complete blood count (CBC), complete metabolic panel (CMP), creatine kinase (CK), antinuclear antibodies (ANA), antineutrophil cytoplasmic antibodies (ANCA) and rheumatoid factor (RF); results were all unremarkable. Inflammatory markers showed CRP of $6.0 \mathrm{mg} / \mathrm{I}$ (normal range $0-10 \mathrm{mg} / \mathrm{l}$ ) and ESR of $30 \mathrm{~mm} / \mathrm{hr}$ (normal range $0-30 \mathrm{~mm} / \mathrm{hr}$ ). The platelet count was normal at $325 \mathrm{~K} / \mu \mathrm{l}$ (normal range $130-430 \mathrm{~K} / \mu \mathrm{l})$. The patient was prescribed a 7-day course of prednisone 20 mg daily. Her symptoms significantly improved thereafter but she deteriorated after stopping steroids. As the clinical presentation was suspicious for GCA, prednisone was restarted and a temporal artery biopsy was performed, with the results confirming the diagnosis of GCA. Steroids were gradually tapered and the patient was prescribed the steroid-sparing medication tocilizumab which has controlled her symptoms very well.

\section{DISCUSSION}

Normal or low levels of inflammatory markers are rare in GCA and PMR, so screening for elevated markers is common, and important for diagnosis. However, due to the wide differential diagnosis, if initial inflammatory markers are normal, diagnosis is often missed or delayed. Complications such as loss of vision and rapid disease progression may occur as a consequence of late diagnosis. Early initiation of high-dose steroids is crucial; they remain the golden standard treatment and can prevent complications, especially blindness ${ }^{[6]}$.

Our patient presented with new onset of proximal myopathy without a clear explanation. Chan et al. found that a combination of CRP and platelet tests may be the best method for diagnosing GCA ${ }^{[7]}$. However, both CRP and platelets were normal in our patient, as was ESR, which if normal makes GCA very unlikely ${ }^{[8]}$. It is estimated that about $4 \%$ of GCA patients may have normal CRP and ESR, but those with normal inflammatory markers may actually have more prominent PMR symptoms and fewer systemic symptoms [8]. Although our patient consistently had a normal CRP, ESR and platelet count throughout the course of the disease, the temporal artery biopsy confirmed the diagnosis of GCA, which supports a body of evidence ${ }^{[9-12]}$ suggesting that normal inflammatory markers should not exclude a diagnosis of GCA and PMR ${ }^{[13]}$. Clinical evaluation for any disease is very important. Normal inflammatory markers should not exclude the possibility of GCA or PMR if the clinical presentation is suggestive, and steroid treatment should be started in order to avoid complications like blindness. Also proper evaluation with advanced diagnostic modalities like temporal artery biopsy (TAB), and radiological diagnostics like temporal ultrasound and/or MRI, should be employed if needed.

By presenting this case of temporal arteritis with normal inflammatory markers, we hope to increase clinician recognition and awareness of the different presentations of this disease. 


\section{REFERENCES}

1. Maz M, Chung SA, Abril A, Langford CA, Gorelik M, Guyatt G, et al. 2021 American College of Rheumatology/Vasculitis Foundation guideline for the management of giant cel arteritis and Takayasu arteritis. Arthritis Rheumatol 2021;73(8):1349-1365.

2. González-Gay MA, Matteson EL, Castañeda S. Polymyalgia rheumatica. Lancet 2017;390(10103):1700-1712.

3. Hunder GG, Bloch DA, Michel BA, Stevens MB, Arend WP, Calabrese LH, et al. The American College of Rheumatology 1990 criteria for the classification of giant cell arteritis Arthritis Rheum 1990;33(8):1122-1128.

4. Turesson C, Börjesson O, Larsson K, Mohammad AJ, Knight A. Swedish Society of Rheumatology 2018 guidelines for investigation, treatment, and follow-up of giant cell arteritis. Scand J Rheumatol 2019;48(4):259-265.

5. Dasgupta B, Cimmino MA, Maradit-Kremers H, Schmidt WA, Schirmer M, Salvarani C, et al. 2012 Provisional classification criteria for polymyalgia rheumatica: a European League Against Rheumatism/American College of Rheumatology collaborative initiative. Ann Rheum Dis 2012;71(4):484-492.

6. Dasgupta B, Borg FA, Hassan N, Alexander L, Barraclough K, Bourke B, et al. BSR and BHPR guidelines for the management of giant cell arteritis. Rheumatology (Oxford) 2010;49(8):1594-1597.

7. Chan FLY, Lester S, Whittle SL, Hill CL. The utility of ESR, CRP and platelets in the diagnosis of GCA. BMC Rheumatol 2019;3(1):14.

8. Smetana GW, Shmerling RH. Does this patient have temporal arteritis? JAMA 2002;287(1):92-101.

9. Cheema MR, Ismaeel SM. Temporal arteritis with erythrocyte sedimentation rate $<50 \mathrm{~mm} / \mathrm{h}$ : a clinical reminder. Clin Interv Aging 2016;11:185-188.

10. Singh R, Sahbudin I, Filer A. New headaches with normal inflammatory markers: an early atypical presentation of giant cell arteritis. BMJ Case Rep 2018;2018:bcr2017223240.

11. Walters B, Lazic D, Ahmed A, Yiin G. Lessons of the month 4: Giant cell arteritis with normal inflammatory markers and isolated oculomotor nerve palsy. Clin Med 2020;20(2):224-226.

12. Salvarani C, Hunder GG. Giant cell arteritis with low erythrocyte sedimentation rate: frequency of occurence in a population-based study. Arthritis Rheum 2001;45(2):140145.

13. Kermani TA, Schmidt J, Crowson CS, Ytterberg SR, Hunder GG, Matteson EL, et al. Utility of erythrocyte sedimentation rate and C-reactive protein for the diagnosis of giant cell arteritis. Semin Arthritis Rheum 2012;41(6):866-871. 